\title{
Exponential Decay Rate of the Perturbed Energy of the Wave Equation with Zero Order Term
}

\author{
Hamchi Ilhem \\ Department of Mathematics, University of Batna, Algeria \\ E-mail: hamchi_ilhem@yahoo.fr \\ Received April 30, 2011; revised May 26, 2011; accepted June 10, 2011
}

\begin{abstract}
In this paper, we consider the wave equation with zero order term. We use the compactness uniqueness argument and some result of I. Lasiecka and D. Tataru in [4] to prove, directly, the exponential decay rate of the perturbed energy.
\end{abstract}

Keywords: Perturbed Energy, Compactness Uniqueness Argument

\section{Introduction}

Consider the boundary feed-back system

$$
\left\{\begin{array}{c}
y_{t t}-\Delta y+q y=0 \text { in } Q, \\
y=0 \text { on } \Sigma_{0}, \\
\frac{\partial y}{\partial v}+b y_{t}=0 \text { on } \Sigma_{1}, \\
y(0)=y_{0}, y_{t}(0)=y_{1} \text { in } \Omega .
\end{array}\right.
$$

Here $\Omega$ is a bounded domain of $\mathbb{R}^{n}\left(n \in \mathbb{N}^{*}\right)$ with smooth boundary $\Gamma$ and $\left\{\Gamma_{0}, \Gamma_{1}\right\}$ is a partition of $\Gamma$ such that $\Gamma_{0} \neq \varnothing$ and $\overline{\Gamma_{0}} \cap \overline{\Gamma_{1}}=\varnothing . \quad T>0$,

$Q=\Omega \times] 0, T\left[, \quad \Sigma_{l}=\Gamma_{l} \times\right] 0, T\left[\quad(l=0,1) . \quad b: \Gamma_{1} \rightarrow \mathbb{R}\right.$ and $q: \Omega \rightarrow \mathbb{R}$ are two positive bounded functions, that is there exists four positive constants $b_{*}, b^{*}, q_{*}, q^{*}$ such that $0<b_{*} \leq b(x) \leq b^{*}$ for all $x \in \Gamma_{1}$, and

$0<q_{*} \leq q(x) \leq q^{*}$ for all $x \in \Omega$.

Fix a point $x_{0}$ of $\mathbb{R}^{n}$. Set $h=h(x)=x-x_{0}$ and $R=\sup \{|h(x)|: x \in \bar{\Omega}\}$. Assume that for some constant $h_{0}>0$ we have

$$
\Gamma_{0}=\{x \in \Gamma: h . v \leq 0\} \text { and } \Gamma_{1}=\left\{x \in \Gamma: h . v \geq h_{0}\right\} .
$$

For all $\varepsilon>0$, we define the perturbed energy of the system (1) by $E^{\varepsilon}(t)=E(t)+\varepsilon \rho(t)$, for all $t \geq 0$. Here $E$ is the usual energy defined by

$$
\begin{aligned}
& E(t)=\frac{1}{2} \int_{\Omega}\left(\left|y_{t}\right|^{2}+q|y|^{2}+|\nabla y|^{2}\right) \mathrm{d} x \text { and } \\
& \rho(t)=\int_{\Omega} y_{t} M y \mathrm{~d} x \text {, where } M y=2 h . \nabla y+(n-1) y .
\end{aligned}
$$

In the case of $q=0, \mathrm{~V}$. Komornick and E. Zuazua in [3] have shown that $E^{\varepsilon}$ decays exponentially. When $q \neq 0$, there is some difficulty to obtain this result since we have a lower order term with respect to the energy in some multiplier estimate (see (3.5) in [3]). The purpose of this paper is to overcome this kind of difficulty, where we prove an useful estimate then by the compactness uniqueness argument we absorb the lower order term. Finally, we employ some result of I. Lasiecka and D. Tataru in [4].

In all this paper, $C$ is a generic positive constant independent of the initial data and it may change from line to line.

\section{Exponential decay rate of $E^{\varepsilon}$}

Using the multiplier method we can show that the energy $E$ of the system (1) is a decreasing function. That is, for all $t \geq 0$, we have

$$
E_{t}(t)=\frac{\mathrm{d} E}{\mathrm{~d} t}=-\int_{\Gamma_{1}} b\left|y_{t}\right|^{2} \mathrm{~d} \Gamma \leq 0 .
$$

The proof of the main theorem involves two lemmas.

Lemma 1 For all two positive constants $T$ and $S$ such that $T-S>T_{0}$, where $T_{0}$ is some sufficiently large positive constant, we have for $\varepsilon$ sufficiently small

$$
\frac{3}{4} C \varepsilon T_{0} E^{\varepsilon}(T) \leq E^{\varepsilon}(S)+C \varepsilon \iint_{S \Omega}^{T}|y|^{2} \mathrm{~d} Q .
$$

Here $\mathrm{d} Q=\mathrm{d} x \mathrm{~d} t$.

Proof. First we have from the definition of $E^{\varepsilon}$

$$
(1-\varepsilon \theta) E(t) \leq E^{\varepsilon}(t) \leq(1+\varepsilon \theta) E(t),
$$

where $\theta$ is the constant verifying 


$$
|\rho(t)|=\left|\int_{\Omega} y_{t} M y \mathrm{~d} x\right| \leq \theta E(t) .
$$

On the other hand, we have (see (3.5) in [3])

$$
E_{t}^{\varepsilon}(t) \leq-C \varepsilon E^{\varepsilon}(t)+C \varepsilon \int_{\Omega}|q y|^{2} \mathrm{~d} x
$$

Integrate over $[S, T]$ use (4) with $\varepsilon$ sufficiently small and by the decreasing of $E$, we find

$$
\begin{aligned}
& -E^{\varepsilon}(S) \leq E^{\varepsilon}(T)-E^{\varepsilon}(S) \\
& \leq-C \varepsilon T_{0} \frac{1-\varepsilon \theta}{1+\varepsilon \theta} E^{\varepsilon}(T)+C \varepsilon \iint_{S \Omega}^{T} \int|y|^{2} \mathrm{~d} Q .
\end{aligned}
$$

Therefore

$$
C \varepsilon T_{0} \frac{1-\varepsilon \theta}{1+\varepsilon \theta} E^{\varepsilon}(T) \leq E^{\varepsilon}(S)+C \varepsilon \int_{S \Omega}^{T} \int|y|^{2} \mathrm{~d} Q .
$$

With $\varepsilon$ sufficiently small we find the desired result.

To absorb the lower order term $\int_{S \Omega}^{T} \int|y|^{2} \mathrm{~d} Q$ from the estimate (3) we apply the compactness uniqueness argument (see for example [1]).

Lemma 2 For $T-S>T_{0}$, where $T_{0}$ is sufficiently large, we have

$$
\int_{S \Omega}^{T} \int|y|^{2} \mathrm{~d} Q \leq C \int_{S \Gamma_{1}}^{T} \int_{1} b\left|y_{t}\right|^{2} \mathrm{~d} \Sigma
$$

where $\mathrm{d} \Sigma=\mathrm{d} \Gamma \mathrm{d} t$.

Proof. First, we have from (3)

$$
E^{\varepsilon}(T) \leq \frac{C}{\varepsilon T_{0}} E^{\varepsilon}(S)+\frac{C}{T_{0}} \iint_{S \Omega}^{T}|y|^{2} \mathrm{~d} Q
$$

with $T_{0}$ sufficiently large, we obtain

$$
E^{\varepsilon}(T) \leq \frac{C}{\varepsilon T_{0}} E^{\varepsilon}(S)+\int_{S \Omega}^{T}|y|^{2} \mathrm{~d} Q .
$$

On the other hand, by (2)

$$
\begin{aligned}
E(S) & =E(T)-\int_{S}^{T} E_{t}(t) \mathrm{d} t=E(T)+\int_{S \Gamma_{1}}^{T} \int b\left|y_{t}\right|^{2} \mathrm{~d} \Sigma \\
& \leq \frac{1}{1-\varepsilon \theta} E^{\varepsilon}(T)+\int_{S \Gamma_{1}}^{T} b\left|y_{t}\right|^{2} \mathrm{~d} \Sigma .
\end{aligned}
$$

Then, with $\varepsilon$ sufficiently small, we find

$$
\begin{aligned}
E^{\varepsilon}(S) & \leq(1+\varepsilon \theta) E(S) \\
& \leq \frac{1+\varepsilon \theta}{1-\varepsilon \theta} E^{\varepsilon}(T)+(1+\varepsilon \theta) \int_{S \Gamma_{1}}^{T} \int b\left|y_{t}\right|^{2} \mathrm{~d} \Sigma \\
& \leq \frac{4}{3} E^{\varepsilon}(T)+2 \iint_{S \Gamma_{1}}^{T} b\left|y_{t}\right|^{2} \mathrm{~d} \Sigma,
\end{aligned}
$$

by (6), we find

$$
E^{\varepsilon}(S) \leq \frac{C}{\varepsilon T_{0}} E^{\varepsilon}(S)+C \int_{S \Omega}^{T} \int|y|^{2} \mathrm{~d} Q+C \int_{S \Gamma_{1}}^{T} \int b\left|y_{t}\right|^{2} \mathrm{~d} \Sigma,
$$

with $T_{0}$ sufficiently large, we find

$$
E^{\varepsilon}(S) \leq C\left(\int_{S \Gamma_{1}}^{T} \int_{1} b\left|y_{t}\right|^{2} \mathrm{~d} \Sigma+\iint_{S \Omega}^{T}|y|^{2} \mathrm{~d} Q\right) .
$$

Now, we come back to the proof of (5).

It is sufficient to prove (see [1]] that, for some $T_{0}$ large enough, we have

$$
\int_{0}^{T_{0}} \int_{\Omega}|y|^{2} \mathrm{~d} Q \leq C \int_{0}^{T_{0}} \int_{\Gamma_{1}} b\left|y_{t}\right|^{2} \mathrm{~d} \Sigma
$$

We argue by contradiction. There exists a sequence of solutions $\left(y_{k}\right)$ of system (1) such that

$$
\lim _{k \rightarrow \infty} \int_{0}^{T_{0}} \int_{\Gamma_{1}} b\left|y_{k t}\right|^{2} \mathrm{~d} \Sigma=0
$$

and

$$
\int_{0}^{T_{0}} \int_{\Omega}\left|y_{k}\right|^{2} \mathrm{~d} Q=1 \text { for all } k,
$$

where $E_{k}$ represents the energy of $y_{k}$.

Let, for all $k, E_{k}^{\varepsilon}(t)=E_{k}(t)+\varepsilon \rho_{k}(t)$, where $\rho_{k}(t)=\int_{\Omega} y_{k t} M y_{k} \mathrm{~d} x$.

If we apply (7) with $E^{\varepsilon}(t)=E_{k}^{\varepsilon}(t), \quad S=0$ and $T=T_{0}$, we obtain by (8), (9) and (4) that $\left(E_{k}(0)\right)$ is bounded and therefore there exists a subsequence $\left(y_{k}\right)$ such that

$$
y_{k} \rightarrow y \text { weakly*in } L^{\infty}\left(0, T_{0} ; H_{\Gamma_{0}}^{1}(\Omega)\right),
$$

and

$$
y_{k} \rightarrow \text { yweaklyin } L^{2}(] 0, T_{0}[\times \Gamma) .
$$

Using (8) and passing to the limit, we obtain

$$
\begin{aligned}
& \left.y_{t t}-\Delta y+q y=0 \text { in }\right] 0, T_{0}[\times \Omega, \\
& y=0 \text { on }] 0, T_{0}\left[\times \Gamma_{0},\right. \\
& \left.\frac{\partial y}{\partial v}=-b y_{t}=0 \text { on }\right] 0, T_{0}\left[\times \Gamma_{1} .\right.
\end{aligned}
$$

Hence we find

(9).

$$
\text { If } y=0 \text { then } \lim _{k \rightarrow \infty} \int_{0}^{T_{0}} \int_{\Omega}\left|y_{k}\right|^{2} \mathrm{~d} Q=0 \text {. This contradicts }
$$

If $y \neq 0$, then $z=y_{t}$ is solution of

$$
\left.z_{t t}-\Delta z+q z=0 \text { in }\right] 0, T_{0}[\times \Omega,
$$




$$
\begin{aligned}
& z=0 \text { on }] 0, T_{0}[\times \Gamma, \\
& \left.\frac{\partial z}{\partial v}=0 \text { on }\right] 0, T_{0}\left[\times \Gamma_{1} .\right.
\end{aligned}
$$

Then, for $T_{0}$ sufficiently large, $z=0$. So, $y$ is a solution of

$$
\begin{aligned}
& -\Delta y+q y=0 \text { in }] 0, T_{0}[\times \Omega, \\
& y=0 \text { on }] 0, T_{0}\left[\times \Gamma_{0},\right. \\
& \left.\frac{\partial y}{\partial v}=0 \text { on }\right] 0, T_{0}\left[\times \Gamma_{1} .\right.
\end{aligned}
$$

If we multiply the first equation by $y$, integrate over $Q$ and use the first Green's formula we find

$$
\int_{0}^{T_{0}} \int_{\Omega}\left(|\nabla y|^{2}+q|y|^{2}\right) \mathrm{d} Q=0,
$$

thus $y=0$.

We give, now, the proof of the main result.

Theorem 3 For any initial data $\left(y_{0}, y_{1}\right) \in D$, the energy perturbed $E^{\varepsilon}$ is exponentially stable.

Proof. If we insert (5) in (3) we find

$$
\frac{3}{4} C \varepsilon T_{0} E^{\varepsilon}(T) \leq E^{\varepsilon}(S)+C \varepsilon \int_{S \Gamma_{1}}^{T} \int_{\Gamma_{1}} b\left|y_{t}\right|^{2} \mathrm{~d} \Sigma,
$$

But

$$
\begin{aligned}
\int_{S \Gamma_{1}}^{T} b\left|y_{t}\right|^{2} \mathrm{~d} \Sigma & =-\int_{S}^{T} E_{t}(t) \mathrm{d} t=E(S)-E(T) \\
& \leq \frac{1}{1-\varepsilon \theta} E^{\varepsilon}(S) .
\end{aligned}
$$

Then

$$
C \varepsilon T_{0} E^{\varepsilon}(T) \leq\left(\frac{C \varepsilon}{1-\varepsilon \theta}+1\right) E^{\varepsilon}(S)
$$

If we choose $T_{0}$ sufficiently large we find $0<r<1$ such that

$$
\frac{1}{r} E^{\varepsilon}(T) \leq E^{\varepsilon}(S),
$$

for all $T-S \geq T_{0}$.

This imply that

$$
E^{\varepsilon}(T) \leq E^{\varepsilon}(S) \text { for all } T-S \geq T_{0} .
$$

If we apply (10) repeatedly on the intervals $\left[m T_{0},(m+1) T_{0}\right], m=0,1, \cdots$, we get

$$
\begin{aligned}
& E^{\varepsilon}\left((m+1) T_{0}\right)+\frac{1-r}{r} E^{\varepsilon}\left((m+1) T_{0}\right) \\
= & \frac{1}{r} E^{\varepsilon}\left((m+1) T_{0}\right) \leq E^{\varepsilon}\left(m T_{0}\right) .
\end{aligned}
$$

Put $p(s)=\frac{1-r}{r} s$ and $s_{m}=E^{\varepsilon}\left(m T_{0}\right)$, to find

$$
s_{m+1}+p\left(s_{m+1}\right) \leq s_{m} .
$$

Using lemma 3.3 in [4] to obtain for all $m$

$$
s_{m} \leq S(m),
$$

where $S(t)$ is the solution of the system

$$
\left\{\begin{array}{c}
S_{t}(t)+(1-r) S(t)=0 \\
S(0)=E^{\varepsilon}(0)
\end{array}\right.
$$

Here we have used $S(t)-[I+p]^{-1}(S(t))=S(t)-$ $\left(I+\frac{1-r}{r} I\right)^{-1}(S(t))=(1-r) S(t)$.

The last system have the solution $S(t)=e^{-(1-r) t} E^{\varepsilon}(0)$, then

$$
E^{\varepsilon}\left(m T_{0}\right) \leq e^{-(1-r) m} E^{\varepsilon}(0) \text { for all } m .
$$

Let $t \geq T_{0}$, then

$$
t=T_{0}+m T_{0}+\tau \text { where } 0 \leq \tau \leq T_{0},
$$

this imply that

$$
t=m T_{0}+\tau^{*} \text { where } T_{0} \leq \tau^{*}=T_{0}+\tau \leq 2 T_{0} .
$$

Then by (11)

$$
E^{\varepsilon}(t)=E^{\varepsilon}\left(m T_{0}+\tau^{*}\right) \leq E^{\varepsilon}\left(m T_{0}\right) .
$$

So

$$
E^{\varepsilon}(t) \leq E^{\varepsilon}\left(m T_{0}\right) \leq e^{-(1-r) \frac{t-\tau^{*}}{T_{0}}} E^{\varepsilon}(0) \leq e^{-(1-r) \frac{t-2 T_{0}}{T_{0}}} E^{\varepsilon}(0) .
$$

Thus

$$
E^{\varepsilon}(t) \leq c^{\prime} E^{\varepsilon}(0) e^{-\omega^{\prime} t} \text { for } t \geq T_{0},
$$

where $c^{\prime}=e^{2(1-r)}$ and $\omega^{\prime}=\frac{1-r}{T_{0}}$.

Remark 4 We can treat exactly in the same way the situation of the second order hyperbolic equation with variable coefficients, linear zero order term and polynomial growth of the nonlinear feedback near the origin. In this case, we use the Riemann geometric approach to handle the case of the variable coefficients principal part (see [2]] to show, directly, that we have an exponential or polynomial decay rate of the perturbed energy functional defined for all $\varepsilon>0$ by

$$
E^{\varepsilon}(t)=E(t)+\varepsilon \rho(t)(E(t))^{\frac{\gamma-1}{2}},
$$

where $\gamma$ depends on the behavior of nonlinear damping at the origin.

\section{References}

[1] S. Feng and X. Feng, "Nonlinear Internal Damping of 
Wave Equations with Variable Coefficients," Acta Mathematica Sinica, Vol. 20, No. 6, 2004, pp. 1057-1072. doi:10.1007/s10114-004-0394-3

[2] Y. Guo and P. F. Yao, "Stabilization of Euler-Bernoulli Plate Equation with Variable Coefficients by Nonlinear Boundary Feedback,” Journal of Mathematical Analysis and Applications, Vol. 317, No. 1, 2006, pp. 50-70. doi:10.1016/j.jmaa.2005.12.006

[3] V. Komornick and E. Zuazua, “A Direct Method for Boundary Stabilization of the Wave Equation,” Journal de Mathématiques Pures et Appliquées, Vol. 69, 1990, pp. 33-54. 